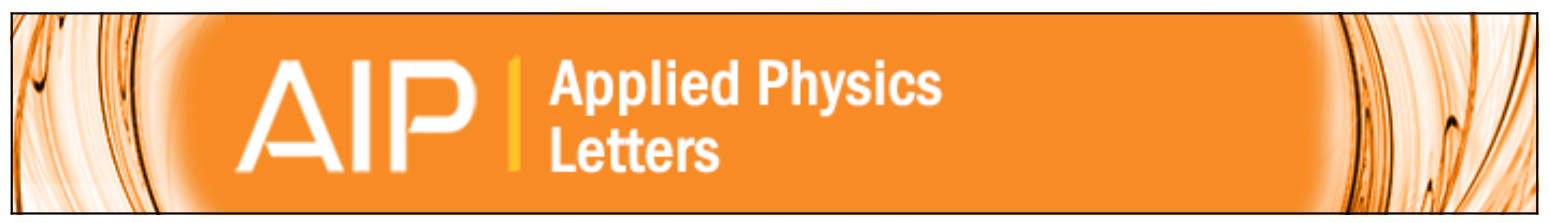

\title{
Optically-addressed two-terminal multicolor photodetector
}

E. H. Steenbergen, M. J. DiNezza, W. H. G. Dettlaff, S. H. Lim, and Y.-H. Zhang

Citation: Applied Physics Letters 97, 161111 (2010); doi: 10.1063/1.3505137

View online: http://dx.doi.org/10.1063/1.3505137

View Table of Contents: http://scitation.aip.org/content/aip/journal/apl/97/16?ver=pdfcov

Published by the AIP Publishing

\section{Articles you may be interested in}

Quantum efficiency investigations of type-II InAs/GaSb midwave infrared superlattice photodetectors

J. Appl. Phys. 116, 043101 (2014); 10.1063/1.4890309

Efficiency enhancement calculations of state-of-the-art solar cells by luminescent layers with spectral shifting, quantum cutting, and quantum tripling function

J. Appl. Phys. 114, 084502 (2013); 10.1063/1.4819237

$1.5 \mu \mathrm{m}$ to $0.87 \mu \mathrm{m}$ optical upconversion using wafer fusion technology

J. Vac. Sci. Technol. A 22, 788 (2004); 10.1116/1.1689300

Demonstration of a $256 \times 256$ middle-wavelength infrared focal plane array based on InGaAs/InGaP quantum dot infrared photodetectors

Appl. Phys. Lett. 84, 2232 (2004); 10.1063/1.1688000

Pixel-less infrared imaging based on the integration of an n-type quantum-well infrared photodetector with a light-emitting diode

Appl. Phys. Lett. 75, 563 (1999); 10.1063/1.124442

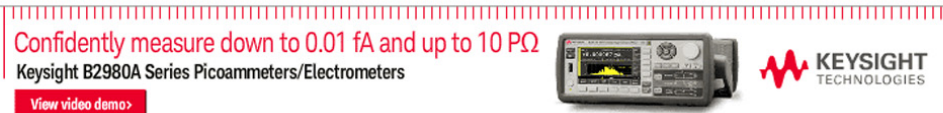




\title{
Optically-addressed two-terminal multicolor photodetector
}

\author{
E. H. Steenbergen, M. J. DiNezza, W. H. G. Dettlaff, S. H. Lim, and Y.-H. Zhang ${ }^{\text {a) }}$ \\ Center for Photonics Innovation and School of Electrical, Computer and Energy Engineering, \\ Arizona State University, Tempe, Arizona 85287, USA
}

(Received 22 September 2010; accepted 2 October 2010; published online 22 October 2010)

\begin{abstract}
A two-terminal multicolor photodetector that is most advantageous for greater than two bands is proposed. This two-terminal design is particularly significant for focal plane arrays as it maximizes the fill factor and simplifies the readout integrated circuits. Individual color detection is realized with appropriate optical biasing. This concept is demonstrated experimentally using a three-color photodetector and biasing light emitting diodes. The measured linear dynamic range is greater than four orders of magnitude, making it a practical device for a broad range of applications. () 2010 American Institute of Physics. [doi:10.1063/1.3505137]
\end{abstract}

Multicolor photodetectors and focal plane arrays (FPAs) are desirable for a variety of applications, including defense, imaging, environmental monitoring, communications, and spectroscopy. ${ }^{1-3}$ Three-terminal monolithic, dual-band photodiodes ${ }^{2}$ and two-terminal, voltage-biasswitchable, back-to-back dual-band photodiodes ${ }^{4,5}$ are examples of existing two-color photodetectors. For FPAs to have more than two colors, additional terminals must be added for each pixel, ${ }^{6-8}$ which greatly complicates the FPA layout and device processing, decreases the fill factor, and increases the readout integrated circuit (ROIC) complexity. ${ }^{6}$ This paper proposes an optically-addressed two-terminal multicolor photodetector that maximizes the fill factor and simplifies the ROIC design. This photodetector makes FPAs with greater than two bands and only two terminals per pixel possible through the use of appropriate optical biasing to realize individual color detection.

The photodetector structure and system operating principles are as follows. The proposed two-terminal photodetector, as shown in Fig. 1, consists of multiple photodiodes with different cutoff wavelengths monolithically connected in series with tunnel diodes between adjacent photodiodes. Due to the series connection, the photodiode with the smallest current dictates the output of the device. To optically-address one of the photodiodes, all of the photodiodes except one, the active detector, are optically biased using a set of light emitting diodes (LEDs) or laser diodes with output wavelengths within the spectral response range of the individual photodiodes. The photodiode's spectral response range spans the energies from the photodiode's band gap to the next highest band gap. The optically-biased photodiodes operate in the photovoltaic mode with their forward voltages determined by the operating current of the entire device. ${ }^{9}$ The optical bias creates a reverse voltage bias on the active photodiode, causing it to operate in the photoconductive mode. The device then has a very small current (the dark current of the active photodiode) when the input signal is not within the active photodiode's spectral response range, and thus the device is a single-color detector with the spectral response of the active photodiode only. Such single-color detection is maintained as long as the photogenerated current due to the signal is smaller than the photogenerated currents from the optically-

${ }^{a)}$ Electronic mail: yhzhang@asu.edu. biased photodiodes. A multiplexing control algorithm can determine which combination of biasing LEDs are on to cycle through the available bands, thus enabling multicolor detection. The integration of multijunction photodetectors and the optical biasing scheme allows the number of detecting bands to increase without raising the number of contacts above two per pixel.

To demonstrate this device concept, a commercial InGaP/InGaAs/Ge triple-junction solar cell $\left(2 \times 2 \mathrm{~cm}^{2}\right)$ is used as the multicolor photodetector because the solar cell structure is almost identical to the proposed multicolor photodetector design. Three LEDs with center wavelengths (470, 780 , and $940 \mathrm{~nm}$ ) within the response ranges of the photodiodes are used as the optical biasing sources. The "dark" current density versus voltage $(J-V)$ curves (Fig. 2) of the three photodiodes are measured with matching light bias photon flux $\left(1.0 \times 10^{16}\right.$ photons $\left./ \mathrm{cm}^{2} / \mathrm{s}\right)$ on the inactive photodiodes and no light bias or signal on the active photodiode. The voltage is across the entire device, not just the active photodiode. The spectral responsivity (Fig. 3) of the active photodiode is also measured with equal optical bias photon flux illuminating the inactive photodiodes. In addition, a $2.1 \mathrm{~V}$ bias across the entire device is used for the Ge photo-

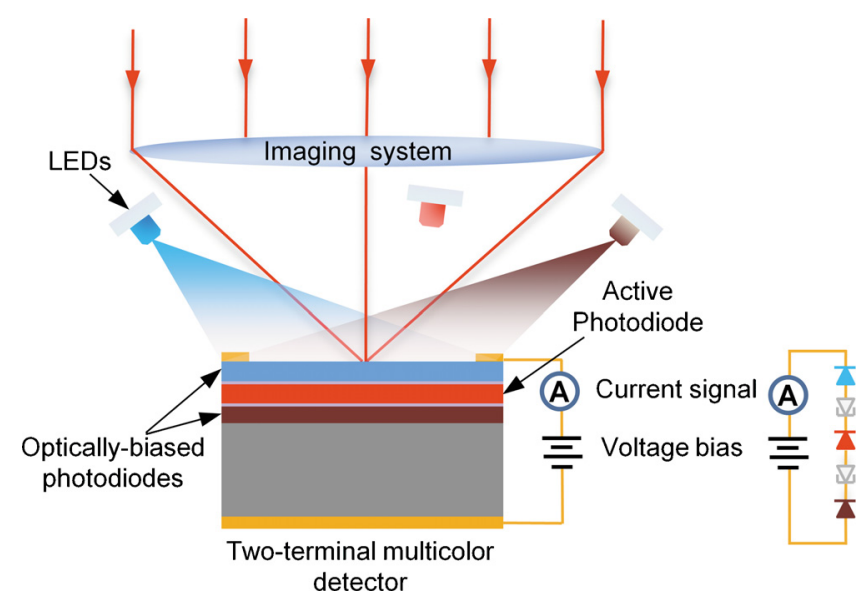

FIG. 1. (Color online) Schematic of the optically addressed, two-terminal, multicolor photodetector. The detector structure consists of multiple photodiodes with different cutoff wavelengths connected in series with tunnel diodes between adjacent photodiodes. The LEDs optically bias the inactive photodiodes in the detector to enable single color detection. 


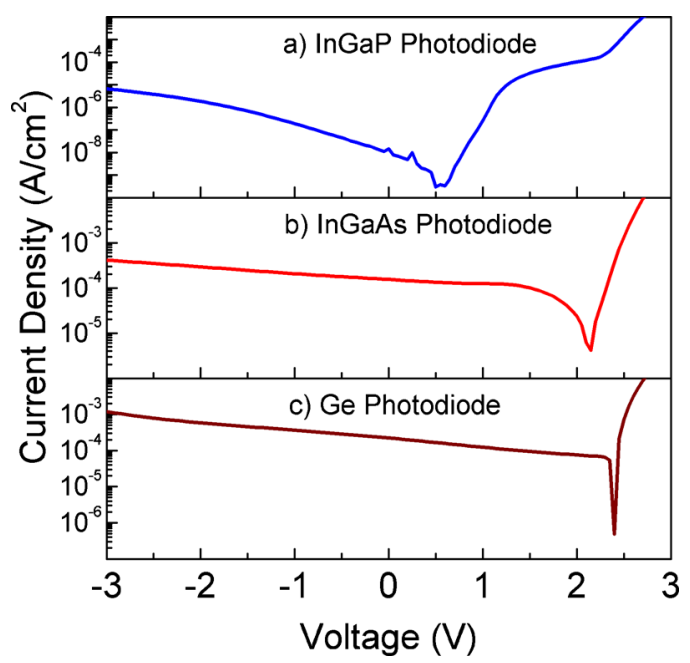

FIG. 2. (Color online) Dark current densities vs. voltage. The $J$-Vs were measured with matching optical bias photon flux on the inactive photodiodes and no input signal to the active photodiode. The voltage is across the entire device, not just the active photodiode.

diode responsivity to reduce measurement artifacts. ${ }^{9,10}$ The spectral response is measured using a Newport QE/IPCE system, with the spectral range limited by the monochromator gratings. The linear dynamic range measurements (Fig. 4) use modulated laser diodes with wavelengths of 405, 780, and $980 \mathrm{~nm}$ as signal sources, while the inactive photodiodes are under equal optical bias flux. Neutral density filters are used to achieve signal intensities over five orders of magnitude, and the resulting current is measured with a lock-in amplifier.

The "dark" current densities versus voltage $(J-V)$ curves are shown in Fig. 2. The forward and reverse bias regions of the individual active photodiodes' $J$ - $V$ s are clearly discernable. The magnitude of the dark current increases as the photodiode's band gap decreases, as expected. The operating point of the active photodiode on the reverse bias portion of its $J-V$ curve depends on the voltage and light bias conditions, which can be selected to minimize the dark current.

The responsivity, as shown in Fig. 3, of the three photodiodes clearly confirms that optical biasing can address one photodiode at a time in a multicolor detector with only two-

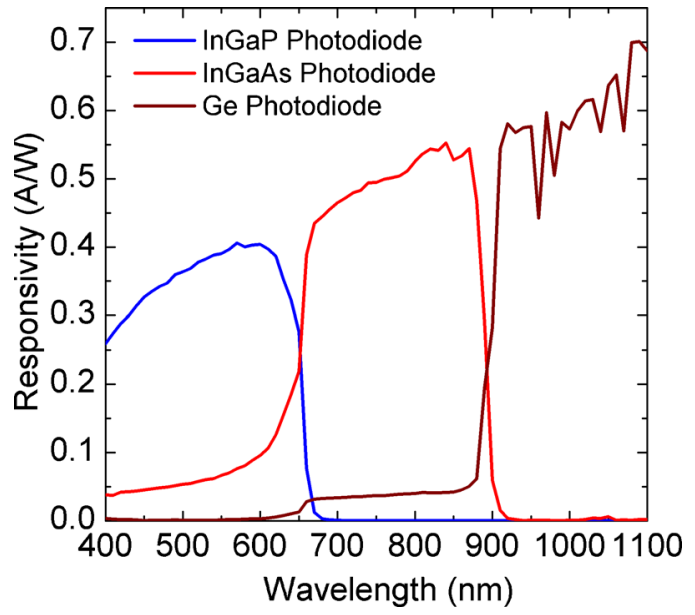

FIG. 3. (Color online) The spectral responsivity curve of each photodiode. The responsivity was measured with matching photon flux on the inactive photodiodes.

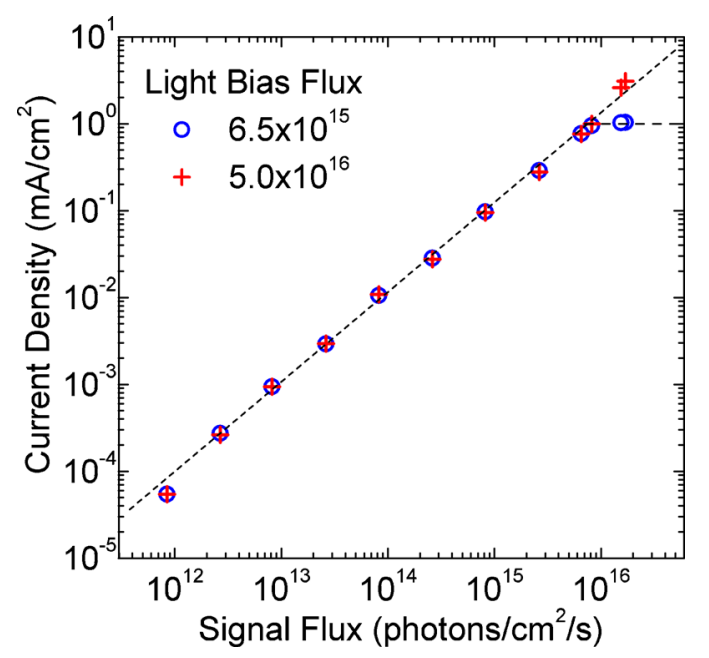

FIG. 4. (Color online) Linear dynamic range of the InGaP photodiode with matching light bias photon flux on the InGaAs and Ge photodiodes. The detector output current saturates after the photogenerated current due to the signal photon flux is larger than the photogenerated current from the light bias photon flux. The dashed lines are guides for the eye.

terminals. When the InGaAs and Ge photodiodes are optically biased, the entire detector response is that of the $\mathrm{InGaP}$ photodiode only, with zero response above $650 \mathrm{~nm}$. The InGaAs photodiode shows a response from 650 to $900 \mathrm{~nm}$, while the Ge photodiode responds at greater than $900 \mathrm{~nm}$. The crosstalk between the InGaP and InGaAs photodiodes and between the InGaAs and Ge photodiodes is a result of (i) luminescence coupling, ${ }^{11,12}$ (ii) photon flux leakage through the InGaP and InGaAs photodiodes, as they may not be optically thick, and (iii) shunts in one or more of the photodiodes. ${ }^{9,10}$ In both cases, the crosstalk responsivity is less than ten percent of the responsivity in the photodiode's intended response range, and this can be further reduced using design modifications.

The linear dynamic range of the InGaP photodiode covers four orders of magnitude, as shown in Fig. 4, under two light bias conditions. The detector current increases linearly as the input signal increases until the photogenerated current due to the signal is larger than the photogenerated current from the light bias. After this point, the detector output saturates due to one of the inactive photodiodes limiting the current. The intensity of the biasing LEDs can limit the upper end of the dynamic range before the detector itself begins to saturate. This is the case in Fig. 4, as increasing the light bias by an order of magnitude allows the detector current to continue to increase. The lower detection limit is determined mainly by the noise of the active photodiode.

In summary, an optically-addressed two-terminal multicolor detector has been proposed, and a two-terminal threecolor detector has been experimentally demonstrated. The measured dark $J-V$, responsivity, and linear dynamic range prove the viability of this optically-addressed two-terminal photodetector concept. Using only two-terminals and incorporating optical biasing allows a FPA with pixels consisting of a large number of monolithic photodiodes to detect, in principle, an almost unlimited number of colors using existing single-color ROICs. Simplified FPA processing and ROIC architectures, along with the increased FPA fill factor, are major advantages of this multicolor detector. 
The authors wish to acknowledge Emcore for donating the triple-junction solar cells, Newport for collaborating on the QE/IPCE Measurement system, Dr. David Zelmon for the use of his equipment at the Air Force Research Laboratory, and Dr. Ding Ding for his original design of Fig. 1. We also acknowledge the financial support of the Science Foundation Arizona fellowship for E.H.S. and M.J.D., the DOD SMART scholarship for E.H.S., and the NSF GRFP for M.J.D. The work done at ASU is partially supported by AFOSR under Grant No. FA9550-10-1-0129.

${ }^{1}$ W. H. Rolls, Electro-Opt. Syst. Des. 9(11), 10 (1977).

${ }^{2}$ J. C. Campbell, T. P. Lee, A. G. Dentai, and C. A. Burrus, Appl. Phys. Lett. 34, 401 (1979).
${ }^{3}$ M. J. Sun, W. S. C. Chang, and C. M. Wolfe, Appl. Opt. 17, 3533 (1978). ${ }^{4}$ E. F. Schulte, U.S. Patent No. 5,113,076 (12 May 1992).

${ }^{5}$ E. R. Blazejewski, J. M. Arias, G. M. Williams, W. McLevige, M. Zandian, and J. Pasko, J. Vac. Sci. Technol. B 10, 1626 (1992).

${ }^{6}$ A. Rogalski, Opto-Electron. Rev. 16, 458 (2008).

${ }^{7}$ K. Kosai, U.S. Patent No. 5,731,621 (24 March 1998).

${ }^{8}$ T. Chu, U.S. Patent No. 5,059,786 (22 October 1991).

${ }^{9}$ M. Meusel, C. Baur, G. Letay, A. W. Bett, W. Warta, and E. Fernandez, Prog. Photovoltaics 11, 499 (2003).

${ }^{10}$ S. H. Lim, K. O'Brien, E. H. Steenbergen, J.-J. Li, and Y.-H. Zhang, Proceedings of the 35th IEEE PVSC (unpublished).

${ }^{11}$ C. Baur, M. Hermle, F. Dimroth, and A. W. Bett, Appl. Phys. Lett. 90, 192109 (2007).

${ }^{12}$ H. Yoon, R. R. King, G. S. Kinsey, S. Kurtz, and D. D. Krut, Proceedings of the 3rd World Conference on Photovoltaic Energy Conversion (Arisumi Printing, Inc., Japan, 2003), p. 745. 